\title{
Asymptotic behaviour of the Euler-Kronecker constant
}

\author{
M. A. Tsfasman \\ To Volodya Drinfeld with friendship and admiration
}

\begin{abstract}
This appendix to the beautiful paper [1] of Ihara puts it in the context of infinite global fields of our papers [2] and [3]. We study the behaviour of Euler-Kronecker constant $\gamma_{K}$ when the discriminant (respectively, the genus) tends to infinity. Results of [2] easily give us good lower bounds on the ratio $\gamma_{K} / \log \sqrt{\left|d_{K}\right|}$. In particular, for number fields, under the generalized Riemann hypothesis we prove

$$
\liminf \frac{\gamma_{K}}{\log \sqrt{\left|d_{K}\right|}} \geq-0.26049 \ldots
$$
\end{abstract}

Then we produce examples of class field towers, showing that

$$
\liminf \frac{\gamma_{K}}{\log \sqrt{\left|d_{K}\right|}} \leq-0.17849 \ldots
$$

\section{Introduction}

Let $K$ be a global field, i.e., a finite algebraic extension either of the field $\mathbb{Q}$ of rational numbers, or of the field of rational functions in one variable over a finite field of constants. Let $\zeta_{K}(s)$ be its zeta-function. Consider its Laurent expansion at $s=1$

$$
\zeta_{K}(s)=c_{-1}(s-1)^{-1}+c_{0}+c_{1}(s-1)+\ldots
$$

In 1] Yasutaka Ihara introduces and studies the constant

$$
\gamma_{K}=c_{0} / c_{-1} .
$$

There are several reasons to study it:

it generalizes the classical Euler constant $\gamma=\gamma_{\mathbb{Q}}$;

for imaginary quadratic fields it is expressed by a beautiful Kronecker limit formula;

for fields with large discriminants (genera) it is astonishingly smaller than the residue $c_{-1}$ itself.

\footnotetext{
${ }^{0}$ Supported in part by the RFBR Grants 02-01-01041, 02-01-22005.
} 
In this appendix we study asymptotic behaviour of this constant when the discriminant (genus) of the field tends to infinity. It is but natural to compare Ihara's results [1] with the methods of infinite zeta-functions developed in [2].

Let $\alpha_{K}=\log \sqrt{\left|d_{K}\right|}$ in the number field case and $\alpha_{K}=\left(g_{K}-1\right) \log q$ in the function field case over $\mathbb{F}_{q}$. In the number field case Ihara shows that

$$
0 \geq \limsup _{K} \frac{\gamma_{K}}{\alpha_{K}} \geq \liminf _{K} \frac{\gamma_{K}}{\alpha_{K}} \geq-1 .
$$

We ameliorate the lower bound to

Theorem 1. Assuming the generalized Riemann hypothesis we have

$\liminf _{K} \frac{\gamma_{K}}{\alpha_{K}} \geq$

$$
-\frac{\log 2+\frac{1}{2} \log 3+\frac{1}{4} \log 5+\frac{1}{6} \log 7}{\frac{1}{\sqrt{2}-1} \log 2+\frac{1}{\sqrt{3}-1} \log 3+\log 5+\frac{1}{\sqrt{7}-1} \log 7+\frac{1}{2}(\gamma+\log 8 \pi)}=-0.26049 \ldots
$$

Remarks. Unconditionally we get $\liminf \gamma_{K} / \alpha_{K} \geq-0.52227 \ldots$

In the function field case using the same method we get $0 \geq \lim \sup \gamma_{K} / \alpha_{K} \geq$ $\liminf \gamma_{K} / \alpha_{K} \geq-(\sqrt{q}+1)^{-1}$, which, of course, coincides with Theorem 2 of Ihara's paper [1].

Let us remark that the upper bound 0 is attained for any asymptotically bad family of global fields, and that the lower bound in the function field case is attained for any asymptotically optimal family (such that the ratio of the number of $\mathbb{F}_{q}$-points to the genus tends to $\sqrt{q}-1$ ), which we know to exist whenever $q$ is a square.

In Section 3 we construct examples of class field towers proving (unconditionally)

\section{Theorem 2.}

$$
\liminf _{K} \gamma_{K} / \alpha_{K} \leq-\frac{2 \log 2+\log 3}{\log \sqrt{5.7 .11 .13 .17 .19 .23 .29 .31 .37}}=-0.17849 \ldots
$$

This slightly ameliorates on the examples given by Ihara in [1.

In the number field case set $\beta_{K}=-\left(\frac{r_{1}}{2}(\gamma+\log 4 \pi)+r_{2}(\gamma+\log 2 \pi)\right)$. If we complete $\gamma_{K}$ by archimedean terms, we get

Theorem 3. Let $\tilde{\gamma}_{K}=\gamma_{K}+\beta_{K}$. Then, under the generalized Riemann hypothesis, we have

$$
\liminf _{K} \frac{\tilde{\gamma}_{K}}{\alpha_{K}} \geq-(\gamma+\log (2 \pi)) /(\gamma+\log (8 \pi))=-0.6353 \ldots
$$

Of course, $\lim \sup \tilde{\gamma}_{K} / \alpha_{K} \leq 0$, and 0 is attained for any asymptotically bad family (i.e., such that all $\phi$ 's vanish). 
The best example we know gives (unconditionally)

Theorem 4.

$$
\liminf _{K} \tilde{\gamma}_{K} / \alpha_{K} \leq-0.5478 \ldots
$$

\section{Bounds}

I am going to consider the asymptotic behaviour of $\gamma_{K}$. We treat the number field case (the same argument in the function field case leads to Theorem 2 of [1]). Let $\left|d_{K}\right|$ tend to infinity. By Lemma 2.2 of [2] any family of fields contains an asymptotically exact subfamily, i.e., such that for any $q$ there exists the limit $\phi_{q}$ of the ratio of the number $\Phi_{q}(K)$ of prime ideals of norm $q$ to the "genus" $\alpha_{K}$, and also the limits $\phi_{\mathbb{R}}$ and $\phi_{\mathbb{C}}$ of the ratios of $r_{1}$ and $r_{2}$ to $\alpha_{K}$. To find $\liminf \gamma_{K} / \alpha_{K}$ and $\liminf \tilde{\gamma}_{K} / \alpha_{K}$ it is enough to find corresponding limits for a given asymptotically exact family, and then to look for their minimal values. In what follows we consider only asymptotically exact families.

Theorem 5. For an asymptotically exact family $\{K\}$ we have

$$
\lim _{K} \frac{\gamma_{K}}{\alpha_{K}}=-\sum \frac{\phi_{q} \log q}{q-1}
$$

where $q$ runs over all prime powers.

Proof. The right-hand side equals $\xi_{\phi}^{0}(1)$, where $\xi_{\phi}^{0}(s)$ is the log-derivative of the infinite zeta-function $\zeta_{\phi}(s)$ of [2]. The corresponding series converges for Re $s \geq 1$ (Proposition 4.2 of [2]). We know ([1], (1.3.3) and (1.3.4)) that

$$
\gamma_{K}=-\lim _{s \rightarrow 1}\left(Z_{K}(s)-\frac{1}{s-1}\right)
$$

where for $\operatorname{Re}(s)>1$

$$
Z_{K}(s)=-\frac{\zeta_{K}^{\prime}}{\zeta_{K}}(s)=\sum_{P, k \geq 1} \frac{\log N(P)}{N(P)^{k s}}=\sum_{q} \Phi_{q}(K) \frac{\log q}{q^{s}-1} .
$$

By the same Proposition 4.2, $\frac{\zeta_{K}^{\prime}}{\zeta_{K}}(s) \rightarrow \xi_{\phi}^{0}(s)$ and hence $\gamma_{K} / \alpha_{K} \rightarrow \xi_{\phi}^{0}(1)$.

Proof of Theorem 1. We have to maximize $\sum \frac{\phi_{q} \log q}{q-1}$ under the conditions:

$\phi_{q} \geq 0$

for any prime $p$ we have $\sum_{m=1}^{\infty} m \phi_{p^{m}} \leq \phi_{\mathbb{R}}+2 \phi_{\mathbb{C}}$;

$\sum_{q} \frac{\phi_{q} \log q}{\sqrt{q}-1}+\phi_{\mathbb{R}}\left(\log 2 \sqrt{2 \pi}+\frac{\pi}{4}+\frac{\gamma}{2}\right)+\phi_{\mathbb{C}}(\log 8 \pi+\gamma) \leq 1$ (Basic Inequality,

GRH-Theorem 3.1 of [2]).

If we put

$$
a_{0}=\log \sqrt{8 \pi}+\frac{\pi}{4}+\frac{\gamma}{2}, a_{1}=\log 8 \pi+\gamma, a_{q}=\frac{\log q}{\sqrt{q}-1}, b_{0}=b_{1}=0, b_{q}=\frac{\log q}{q-1},
$$


we are under conditions (1)-(4) and (i)-(iv) of Section 8 of [2]

Theorem 1 is now straightforward from Proposition 8.3 of [2]. Indeed, the maximum is attained for $\phi_{p^{m}}=0$ for $m>1, \phi_{\mathbb{R}}=0$, and $\phi_{2}=\phi_{3}=\phi_{5}=$ $\phi_{7}=2 \phi_{\mathbb{C}}$ (calculation shows that starting from $p^{\prime}=11$ the last inequality of Proposition 8.3 is violated).

Proof of Theorem 3. It is much easier. Since in this case all coefficients are positive and the ratio of the coefficient of the function we maximize to the corresponding coefficient of the Basic Inequality is maximal for $\phi_{\mathbb{C}}$, the maximum is attained when all $\phi$ 's vanish, except for $\phi_{\mathbb{C}}$.

Remarks. If we want unconditional results, then instead of the Basic inequality we have to use Proposition 3.1 of [2]

$$
2 \sum_{q} \phi_{q} \log q \sum_{m=1}^{\infty} \frac{1}{q^{m}+1}+\phi_{\mathbb{R}}(\gamma / 2+1 / 2+\log 2 \sqrt{\pi})+\phi_{\mathbb{C}}(\gamma+\log 4 \pi) \leq 1
$$

For $\tilde{\gamma}_{K} / \alpha_{K}$ one easily gets

$$
\liminf \frac{\tilde{\gamma}_{K}}{\alpha_{K}} \geq-\frac{\gamma+\log (2 \pi)}{\gamma+\log (4 \pi)}=-0.7770 \ldots
$$

The calculation for $\gamma_{K} / \alpha_{K}$ is more tricky, the last condition of Proposition 8.3 is not violated until very large primes. Changing the coefficients by the first term $(q+1)^{-1}$, Zykin [5] gets

$$
\liminf \frac{\gamma_{K}}{\alpha_{K}} \geq-0.52227 \ldots
$$

Note that (for an asymptotically exact family) $1+\tilde{\gamma}_{K} / \alpha_{K}$ is just the value at 1 of the $\log$-derivative $\xi(s)$ of the completed infinite zeta-function $\tilde{\zeta}(s)$ of $[2$.

\section{$3 \quad$ Examples}

Let us bound liminf $\gamma_{K} / \alpha_{K}$ from above. To do this one should provide some examples of families. The easiest is, just as in Section 9 of [2, to produce quadratic fields having infinite class field towers with prescribed splitting. The proof of Theorem 1 suggests that we should look for towers of totally complex fields, where $2,3,5$ and 7 are totally split. This is however imprecise, because the sum of Proposition 8.3 varies but slightly when we change $p_{0}$. Therefore, I also look at the cases when 2, 3, 5, 7 and 11 are split, and when only 2, 3 and 5 are split, or even only 2 and 3 . This leads to a slight amelioration on (1.6.30) of [1].

Each of the following fields has an infinite 2-class field tower with prescribed splitting (just apply Theorem 9.1 of [2]), and Theorem 5 gives the following list. For $\mathbb{Q}(\sqrt{11.13 .17 .19 .23 .29 .31 .37 .41 .43 .47 .53 .59 .61 .67})$ (the example of Thm.9.4 of [2] $\mathbb{R}, 2,3,5,7$ totally split, we get $\liminf \gamma_{K} / \alpha_{K} \leq-0.1515 \ldots$ 
For $\mathbb{Q}(\sqrt{-13.17 .19 .23 .29 .31 .37 .41 .43 .47 .53 .59 .61 .73 .79})$ (the example of Theorem 9.5 of [2]) with $2,3,5,7$, and 11 split we get $-0.1635 \ldots$

For $\mathbb{Q}(\sqrt{-7.11 .13 .17 .19 .23 .29 .31 .37 .41 .43 .79})$ with $2,3,5$ split we get $-0.1727 \ldots$ For $\mathbb{Q}(\sqrt{-7.11 .13 .17 .19 .23 .29 .31 .37 .41 .47 .59})$ with $2,3,5$ split we get $-0.1737 \ldots$ An even better example is found by Zykin $[5]: \mathbb{Q}(\sqrt{-5.7 .11 .13 .17 .19 .23 .29 .31 .37})$ with 2 and 3 split gives us $-0.17849 \ldots$ This proves Theorem 2.

For liminf $\tilde{\gamma}_{K} / \alpha_{K}$ the Martinet field $\mathbb{Q}\left(\cos \frac{2 \pi}{11}, \sqrt{2}, \sqrt{-23}\right)$ (see Theorem 9.2 of [2]) gives $-0.5336 \ldots$ The best Hajir-Maire example (see 4, Section 3.2) gives $\lim \inf \tilde{\gamma}_{K} / \alpha_{K} \leq-0.5478 \ldots$ This proves Theorem 4 .

Acknowledgements. I am grateful to Professor Yasutaka Ihara for letting me know his results prior to publication and for a useful e-mail discussion, and to my student Alexei Zykin for many fruitful discussions and for computer verification of my calculations.

\section{References}

[1] Y. Ihara. On the Euler-Kronecker constants of global fields and primes with small norms. - This volume.

[2] M.A. Tsfasman, S.G. Vlăduţ. Infinite Global Fields and the Generalized Brauer-Siegel Theorem. Moscow Math. J., 2002, v.2, n.2, pp.329-402.

[3] M.A. Tsfasman, S.G. Vlăduţ. Asymptotic properties of zeta-functions. J. Math. Sciences (New York), 1997, v.84, n.5, pp.1445-1467.

[4] F. Hajir, C. Maire. Tamely ramified towers and discriminant bounds for number fields II. J. Symbolic Computation, 2002, v.33 , no.4, pp. 415-423.

[5] A.Zykin. Private communication.

Poncelet Laboratory (UMI 2615 of CNRS and the Independent University of Moscow) 11 Bolshoi Vlasievskii per., Moscow 119002, Russia; and

Institut de Mathématiques de Luminy, Luminy Case 907, Marseille 13288, France

E-mail: tsfasman@iml.univ-mrs.fr 